\title{
Author Correction: Research Priorities for the Intersection of Alcohol and HIV/AIDS in Low and Middle Income Countries: A Priority Setting Exercise
}

\author{
Sarah Gordon $^{1} \cdot$ Mary Jane Rotheram-Borus $^{2,5} \cdot$ Sarah Skeen $^{1} \cdot$ Charles Parry $^{3} \cdot$ Kendall Bryant $^{4} \cdot$ Mark Tomlinson $^{1}$
}

Published online: 11 December 2017

(c) Springer Science+Business Media, LLC, part of Springer Nature 2017

\section{Author Correction: \\ AIDS Behav (2017) 21:262-273 \\ https://doi.org/10.1007/s10461-017-1921-4}

The original version of this article unfortunately contained an error in the co-authors name.
The co-author names should be Sarah Gordon and Charles Parry instead of Sara Gordon and Charles Perry.

The original article can be found online at https://doi. org/10.1007/s10461-017-1921-4.

Mary Jane Rotheram-Borus

cchpublications@mednet.ucla.edu

1 The Department of Psychology, Stellenbosch University, Private Bag X1, Matieland 7602, South Africa

2 Department of Psychiatry and Biobehavioral Sciences, Semel Institute, University of California at Los Angeles, 760 Westwood Plaza, Los Angeles, CA 90024, USA

3 South African Medical Research Council, Francie van Zijl Drive, Parow Valley, Cape Town, South Africa

4 National Institute of Alcohol Abuse and Alcoholism, 5635 Fishers Lane, Bethesda, MD 20892-7003, USA

5 Global Center for Children and Families, Semel Institute and the Department of Psychiatry, University of California at Los Angeles, 10920 Wilshire Boulevard, Suite 350, Los Angeles, CA 90024-6521, USA 